Published in final edited form as:

Tob Control. 2017 December ; 26(E2): e117-e126. doi:10.1136/tobaccocontrol-2016-053462.

\title{
Electronic cigarette use among US adults in the Population Assessment of Tobacco and Health (PATH) Study, 2013-2014
}

\author{
Blair N Coleman ${ }^{1}$, Brian Rostron ${ }^{1}$, Sarah E Johnson ${ }^{1}$, Bridget K Ambrose ${ }^{1}$, Jennifer \\ Pearson $^{2,3}$, Cassandra A Stanton ${ }^{4,5}$, Baoguang Wang ${ }^{1}$, Cristine Delnevo ${ }^{6}$, Maansi Bansal- \\ Travers $^{7}$, Heather L Kimmel ${ }^{8}$, Maciej L Goniewicz ${ }^{7}$, Raymond Niaura ${ }^{2,3}$, David Abrams ${ }^{2,3}$, \\ Kevin P Conway ${ }^{8}$, Nicolette Borek ${ }^{1}$, Wilson M Compton ${ }^{8}$, Andrew Hyland ${ }^{7}$ \\ ${ }^{1}$ Center for Tobacco Products, US Food and Drug Administration, Silver Spring, Maryland, USA \\ ${ }^{2}$ Schroeder Institute for Tobacco Research and Policy Studies, Truth Initiative, Washington, \\ District of Columbia, USA \\ ${ }^{3}$ Johns Hopkins Bloomberg School of Public Health, Baltimore, Maryland, USA \\ ${ }^{4}$ Westat Inc, Rockville, Maryland, USA \\ ${ }^{5}$ Department of Oncology, Lombardi Comprehensive Cancer Center, Georgetown University \\ Medical Center, Washington, DC, USA \\ ${ }^{6}$ Center for Tobacco Studies, School of Public Health, Rutgers University, New Brunswick, New \\ Jersey, USA \\ ${ }^{7}$ Roswell Park Cancer Institute, Buffalo, New York, USA \\ ${ }^{8}$ National Institute on Drug Abuse, National Institutes of Health, U.S. Department of Health and \\ Human Services, Bethesda, Maryland, USA
}

\section{Abstract \\ Background-Electronic cigarette (e-cigarette) use in the USA is increasing. As such, it is critical to understand who uses e-cigarettes, how e-cigarettes are used and what types of products are prevalent. This study assesses patterns of current e-cigarette use among daily and non-daily adult users in the 2013-2014 Population Assessment of Tobacco and Health (PATH) Study.}

\footnotetext{
Correspondence to: Dr Blair N Coleman, Division of Population Health Science, Office of Science, Center for Tobacco Products, US Food and Drug Administration, 10903 New Hampshire Avenue, Silver Spring, MD 20903-1058, USA; Blair.Coleman@fda.hhs.gov. Contributors BNC designed the study and directed its implementation. BR conducted the data analysis, including quality assurance and control. SEJ, BKA, JP, CAS, MB-T, HLK, MLG, RN, DA, KPC, NB, WMC and AH contributed to the conceptual design of the study and assisted with drafting of the manuscript (eg, preparing the literature review, identifying key findings and interpretation of study findings in the Discussion section). All coauthors approved the final version of the manuscript prior to submission.

Publisher's Disclaimer: Disclaimer This publication represents the views of the authors and does not necessarily represent the views of the US Food and Drug Administration, the National Institute on Drug Abuse of the US Department of Health and Human Services. Competing interests MLG received research grant from Pfizer and served as a member of advisory board to J\&J, manufacturers of smoking cessation medications. RN served as an expert witness for plaintiff versus tobacco companies. WMC reports long-term stock ownership in General Electric, 3M Companies, and Pfizer unrelated to the contentof this paper. No additional financial disclosures were reported by the authors of this paper.

Ethics approval Westat's Institutional Review Board approved the study design and protocol, and the Office of Management and Budget approved the data collection.

Provenance and peer review Not commissioned; externally peer reviewed.
} 
Methods-We examined the proportion of current adult e-cigarette users $(\mathrm{n}=3642)$ reporting infrequent use (use on 'some days' and use on 0-2 of the past 30 days), moderate use (use on 'some days' and use on $>2$ of the past 30 days) and daily use. We examined demographic characteristics, use of other tobacco products and e-cigarette product characteristics overall and by use category. Adjusted prevalence ratios (aPRs) were calculated using Poisson regression to assess correlates of daily e-cigarette use.

Results-Among the $5.5 \%$ of adult current e-cigarette users in the PATH Study, $42.2 \%$ reported infrequent use, $36.5 \%$ reported moderate use and $21.3 \%$ reported daily use. Cigarette smokers who quit in the past year were more likely to report daily e-cigarette use, compared with current smokers ( $\mathrm{aPR}=3.21,95 \% \mathrm{CI}=2.75$ to 3.76). Those who reported using rechargeable or refillable devices were more likely to report daily use compared with those who did not use these devices ( $\mathrm{aPR}=1.95,95 \% \mathrm{CI}=1.44$ to 2.65 and $\mathrm{aPR}=2.10,95 \% \mathrm{CI}=1.75$ to 2.52 , respectively).

Conclusions-The majority of e-cigarette users in this study reported less than daily use. Compared with non-daily use, daily use was associated with being a former smoker; however, cross-sectional data limits our ability to establish the temporality or directionality of such associations.

\section{INTRODUCTION}

Electronic cigarettes (e-cigarettes) are battery-operated devices typically designed to deliver nicotine and other additives to users in an aerosol form. ${ }^{1}$ Ever and current use of e-cigarettes among US adults has increased in recent years ${ }^{2-4}$ and is associated with current or former cigarette smoking. Research indicates that prevalence of ever and current e-cigarette use is highest among recent former and current smokers. ${ }^{235-7}$ The 2015 National Health Interview Survey (NHIS) found that $22 \%$ of recent former cigarette smokers (quit <1 year ago), $2.3 \%$ of long-term former smokers (quit >12 months ago) and $0.4 \%$ of never-smokers were everyday or someday e-cigarette users. ${ }^{5}$ In another NHIS study, daily use was also highest among former smokers who recently quit ( $<1$ year) smoking (13.0\%) and compared with daily cigarette smokers, those who recently quit smoking were four times as likely to be daily e-cigarette users. ${ }^{6}$

On 5 May 2016, the US Food and Drug Administration extended its jurisdiction to all tobacco products, including e-cigarettes, which gives the agency authority over the manufacturing, marketing and distribution of e-cigarettes. Given this new regulatory authority, a better understanding of who uses e-cigarettes, how e-cigarettes are used (including use frequency and use in the context of other product use) and what types of products are prevalent is critical to assess the impact e-cigarette products will have on population health. For instance, there is cause for public health concern if e-cigarettes promote uptake of smoking by those who would otherwise be non-users of any tobacco, delay or deter smoking cessation in those who would otherwise have quit or encourage relapse to smoking among former smokers. On the other hand, if current cigarette smokers who are otherwise unwilling or unable to quit tobacco use switch completely from cigarette smoking to e-cigarette use, a public health benefit may result. ${ }^{89}$ Although some studies suggest e-cigarettes may help some smokers quit, ${ }^{1011}$ the extent to which cigarette smokers turn to e-cigarettes to augment their continued cigarette smoking, rather than as a cessation 
aid, remains unclear. To date, US national studies have typically stratified ever or current ecigarette use by demographic characteristics or cigarette smoking status and have not reported detailed use patterns, other tobacco product use or reasons for use in the context of their cigarette smoking status.

Understanding the patterns and trends of e-cigarette use over time requires careful consideration of how use is defined. Whereas established conventions exist for defining current use of traditional cigarettes (ie, 100 lifetime cigarette threshold, currently use every day or some days), definitions for e-cigarette use have varied. Definite 'current' e-cigarette use range from any use in the past 30 days, ${ }^{212-14}$ to 'someday' or 'everyday' use, ${ }^{4615-18}$ to other definitions of current use. ${ }^{1619-21}$ As e-cigarette use has grown, researchers have called for more nimble ways to ascertain use patterns. ${ }^{22}$ Defining any e-cigarette use in the past 30 days as current use may conflate recent initiates and casual experimenters with habitual users $^{23}$; these groups may differ in their reasons for e-cigarette use, tobacco use status and likelihood of continuing e-cigarette use. ${ }^{1718}$ Furthermore, patterns of e-cigarette use differ across users and non-users of other tobacco products and across age groups; for example, although researchers found that e-cigarette ever use was most common among current cigarette users and young adults, daily use was most common among recent former smokers and older adults. ${ }^{6}$

This study analyses the 2013-2014 Wave 1 Survey data from the Population Assessment of Tobacco and Health (PATH) Study to assess prevalence and patterns of current e-cigarette use among daily and non-daily adult users. The PATH Study provides a unique opportunity to characterise e-cigarette users in greater detail than has been presented in US national cross-sectional surveys to date. Whereas national surveys generally measure ever use, frequency of use and use of non-tobacco flavoured e-cigarettes, the PATH Study extends existing surveys by collecting detailed product information (eg, device characteristics), purchasing behaviour and motivations for use. This study examines characteristics of daily versus non-daily e-cigarette users (and further by 'infrequent' and 'moderate' non-daily users), as well as indices reflecting use patterns, product characteristics and reasons for use.

\section{METHODS}

\section{Data source}

Data are from Wave 1 Survey of the PATH Study conducted from 12 September 2013 to 15 December 2014. The PATH Study used audio-computer assisted self-interviews (ACASI) available in English and Spanish to collect information on tobacco use and health in a nationally representative, longitudinal cohort study of 45971 civilian, non-institutionalised adults and youth in the USA, ages $\geq 12$ years. This analysis draws from the 32320 adult interviews (ages $\geq 18$ years). Recruitment employed address-based, area-probability sampling, using an in-person household screener to select youths and adults. Adult tobacco users, young adults ages 18-24 and African-Americans were oversampled relative to population proportions. Among households that were screened (weighted household screener rate $=54 \%$ ), the overall weighted response rate was $74.0 \%$ for the adult interview. Further details regarding the PATH Study design and methods are published elsewhere ${ }^{24}$ and in the User Guide to the PATH Study restricted use files, available at http://doi.org/ 10.3886/ 
ICPSR36231. Westat's (the study contractor) Institutional Review Board approved the study design and protocol, and the Office of Management and Budget approved the data collection.

\section{E-cigarette use}

The e-cigarette items in the PATH Study questionnaire displayed a preamble as well as images of e-cigarette devices, cartridges and e-liquid to help respondents identify which products they used. The preamble presented to respondents reads: 'The next questions are about electronic cigarettes, often called e-cigarettes. E-cigarettes look like regular cigarettes, but are battery-powered and produce vapor instead of smoke. There are many types of ecigarettes. Some common brands include NJOY, Blu and Smoking Everywhere'. The questionnaire design allowed for flexibility in the definition of e-cigarette use. Respondents were asked, "Have you ever seen or heard" of an e-cigarette, and then, "Have you ever used an e-cigarette even one or two times?" Individuals who reported ever using an e-cigarette were asked, "Do you now use e-cigarettes...", with response options being every day, some days or 'not at all'. Those who reported use on some days were subsequently asked, "On how many of the past 30 days did you use an e-cigarette?" and selected a number from 0 to 30. Using these survey questions, we classified e-cigarette users based on both self-reported frequency of use and the number of days of use (out of the past 30 days). Infrequent users were defined as those reporting e-cigarette use on some days and on 0-2 of the past 30 days; moderate users were defined as those reporting use on some days and $>2$ of the past 30 days; and daily users were defined as those reporting use of e-cigarettes every day. Two days out of the past 30 was the cut point for infrequent and moderate use because it was the median value for non-daily e-cigarette users. Non-daily users who did not report the number of days used were included in the overall and non-daily user groups but not the more specific infrequent or moderate use subgroups.

All e-cigarette users were asked age of first use, whether their first e-cigarette was flavoured with a non-tobacco flavour (ie, menthol, mint, clove, spice, candy, fruit, chocolate, alcohol and other sweets), whether they used e-cigarettes as a way of cutting down on cigarettes, the number of lifetime cartridges or disposable e-cigarettes consumed and whether the ecigarette usually used was rechargeable and/or refillable. Users of rechargeable e-cigarettes were asked if their device used cartridges. (See http://www.icpsr.umich.edu/icpsrweb/ NAHDAP/studies/36231 for the PATH Study Adult Interview.)

Respondents were also asked whether they ever fairly regularly used e-cigarettes to identify established users who were routed through the questionnaire to answer questions regarding preferred brands, additional device characteristics and purchasing behaviours. All established users (ie, those who met the 'fairly regularly' threshold) were asked their regular brand of e-cigarette, if this brand contained non-tobacco flavourings, if their usual ecigarette contained nicotine, how they usually bought e-cigarettes (ie, in person, via the internet and by telephone) and where e-cigarettes were purchased if in person (ie, convenience store or gas station, smoke shop, tobacco specialty shop and mall kiosk). 


\section{Other tobacco product use characteristics}

Cigarette smoking status was classified as: (1) current smoker, who reported lifetime smoking of $\geq 100$ cigarettes and currently smoking cigarettes either some days or every day; (2) recent quitter, who reported having smoked $\geq 100$ cigarettes, currently not smoking at all and having completely quit smoking within the past year; (3) long-term former smoker, who reported having smoked $\geq 100$ cigarettes, currently not smoking at all and having completely quit smoking more than a year ago; and (4) never-established smoker, who reported not having smoked $\geq 100$ cigarettes $^{\mathrm{i}}$ (hereafter referred to as 'never-smoker'). Respondents were also asked about current use of combusted tobacco products other than cigarettes (ie, filtered cigars, cigarillos, traditional cigars, pipes and hookah) and non-combusted products (ie, smokeless tobacco, snus pouches and dissolvable tobacco) every day or some days.

\section{Reasons for e-cigarette use}

Respondents reporting everyday or someday use were shown a randomised set of 13 potential reasons for use and asked to indicate which reasons they endorsed (see table 1 for full set of stated reasons). Stated reasons ranged from affordability to presence of flavours, and many were relative to cigarette smoking, such as "They might be less harmful to me than cigarette". Responses were analysed by cigarette smoking status (current, recent and long-term former and never-smoker).

\section{Demographic characteristics}

Race/ethnicity was categorised as Hispanic of any race and non-Hispanic white, AfricanAmerican or Black, Asian, American-Indian or Alaskan Native and other or multirace. Socioeconomic status was assessed by the ratio of self-reported family income to the US Federal Poverty Level (FPL) (http://healthcare.gov/glossary/federal-poverty-level-FPL/) and categorised as $<100 \%, 100 \%-199 \%$ and $\geq 200 \%$ of the FPL.

\section{Statistical analysis}

Descriptive statistics of e-cigarette users, patterns of use and product characteristics were stratified by demographic and tobacco use characteristics. Unadjusted and adjusted prevalence ratios were calculated using Poisson regression ${ }^{25}$ to examine the association between everyday versus someday e-cigarette use (including both infrequent and moderate users) and demographic, tobacco use and product characteristics. Analyses were conducted from 2015 to 2016 using SAS version 9.3 with Poisson regression performed with Stata version 12.1. All analyses were conducted using replicate weights and balanced repeated replication methods to account for the PATH Study's complex survey design. Differences in characteristics between groups and subgroups were evaluated using Rao-Scott $\chi^{2}$ tests with statistical significance being $\mathrm{p}<0.05$. Prevalence estimates derived from denominators of less than 50 and those with a relative SE greater than $30 \%$ were suppressed. ${ }^{26}$

\section{Missing data}

Relatively few e-cigarette users had missing data for variables in this study, usually less than $2 \%$, and these users were not included in relevant analyses. In terms of descriptive statistics, $8.0 \%$ of e-cigarettes users did not have income information and $2.0 \%$ did not have 
information on sexual orientation and identity; $2.8 \%$ were missing some information on other combusted product use, and 3.5\% of everyday established users reported 'don't know' or 'refused' regarding the number of e-cigarettes or cartridges used per day. Lastly, $6.6 \%$ of established e-cigarette users who reported owning an e-cigarette did not identify its brand. Of the 3642 current e-cigarette users, 3373 had information for all of the covariates in the regression analysis and were included in this analysis.

\section{RESULTS}

\section{Demographic characteristics and tobacco use status}

The prevalence of current (everyday or someday) e-cigarette use among adults in the PATH Study during Wave 1 Survey was 5.5\% (95\% CI=5.3 to 5.8) (data not shown). Among all current e-cigarette users (unweighted $\mathrm{n}=3642$ ), $42.2 \%$ reported infrequent use, $36.5 \%$ reported moderate use and $21.3 \%$ reported daily use of e-cigarettes. Among those who reported current use of e-cigarettes, $71.0 \%$ identified as non-Hispanic Whites, 53.5\% were men and 52.0\% reported some college education or higher (table 2). Bivariate analyses indicated statistically significant differences by e-cigarette use status for race/ethnicity, age group and income.

Nearly $70 \%$ of all current e-cigarette users reported current cigarette smoking; however, infrequent users were more likely to report current cigarette smoking (76.9\%) compared with moderate or daily users $(73.2 \%(\mathrm{p}=0.046)$ and $49.6 \%(\mathrm{p}<0.0001)$, respectively) (table 2). Infrequent users were also more likely to report other combusted product use (cigars, hookah or pipes) compared with moderate or daily users ( $44.8 \%$ vs $40.8 \%$ ( $\mathrm{p}=0.047)$ and $25.4 \%(\mathrm{p}<0.0001))$. Conversely, daily users were more likely to report recently quitting cigarette smoking compared with moderate or infrequent users $(27.2 \%$ vs $5.2 \%(\mathrm{p}<0.0001)$ and $2.1 \%(\mathrm{p}<0.0001))$ and were also more likely to report being a long-term former cigarette smoker $(14.7 \%)$ compared with moderate or infrequent users $(4.1 \%(\mathrm{p}<0.0001)$ and $2.7 \%$ $(\mathrm{p}<0.0001))$.

\section{Patterns of and reasons for e-cigarette use}

As shown in table 3, patterns of use and device characteristics varied considerably by current e-cigarette use status. Nearly $90 \%$ of daily e-cigarette users reported using e-cigarettes as a way of cutting down on cigarette smoking compared with $81.8 \%(\mathrm{p}=0.001)$ of moderate users and $58.2 \%(p<0.0001)$ of infrequent users. In terms of e-cigarette device characteristics, daily e-cigarette users were more likely to report using rechargeable products (91.6\%) compared with moderate $(78.0 \%, \mathrm{p}<0.0001)$ or infrequent $(58.6 \%, \mathrm{p}<0.0001)$ users and more likely to report using refillable e-cigarette products (73.6\%) compared with moderate $(51.4 \%, \mathrm{p}<0.0001)$ or infrequent $(32.4 \%, \mathrm{p}<0.0001)$ users. Among those who reported using a rechargeable e-cigarette product, infrequent users were more likely to report use of cartridges $(71.0 \%)$ compared with moderate $(61.5 \%, \mathrm{p}=0.0001)$ or daily $(42.3 \%$, $\mathrm{p}<0.0001)$ users.

Overall, the most frequently endorsed reasons (table 1) for using e-cigarettes included that ecigarettes "might be less harmful to people around me than cigarettes", "might be less 
harmful to me than cigarettes", and "e-cigarettes [can be used] at times when or in places where smoking cigarettes isn't allowed". Several reasons for use differed by cigarette smoking status; for instance, never-smokers were more likely to endorse appealing flavours as a reason for e-cigarette use $(75.3 \%)$ compared with current $(63.7 \%, \mathrm{p}<0.0001)$ and former $(60.1 \%, \mathrm{p}<0.0001)$ smokers. Additionally, never-smokers were more likely to endorse they like socialising while using e-cigarettes $(52.0 \%)$ compared with current $(37.3 \%, \mathrm{p}<0.0001)$ and former $(37.4 \%, \mathrm{p}<0.0001)$ smokers. Among former cigarette smokers, the most frequently endorsed reasons for using e-cigarettes included "might be less harmful to me than cigarettes" $(91.8 \%)$, relative harm to others $(86.3 \%)$ and because "e-cigarettes don't smell" $(80.1 \%)$, whereas current smokers most frequently endorsed using e-cigarettes for reasons relating to relative harm to themselves or others $(80.5 \%$ and $85.1 \%$, respectively) and "use at times or in places where smoking isn't allowed" $(83.5 \%)$.

\section{Factors associated with daily versus non-daily e-cigarette use}

Adjusted Poisson regression analysis indicated sex, age group, cigarette smoking status, current use of other combusted tobacco products and device characteristics were significantly associated with daily versus non-daily e-cigarette use in the adjusted model (table 4). Compared with young adults aged 18-24 years, adults aged 55-64 and 65+ years were more likely to be daily e-cigarette users ( $\mathrm{aPR}=1.44,95 \% \mathrm{CI}=1.11$ to 1.87 and $\mathrm{aPR}=1.51,95 \% \mathrm{CI}=1.13$ to 2.01 , respectively). A strong association was identified between cigarette smoking status and daily use of e-cigarettes, such that recently quitting cigarette smoking (vs current smoking) was strongly associated with daily e-cigarette use (aPR=3.21, $95 \% \mathrm{CI}=2.75$ to 3.76 ). In addition, being a long-term former smoker (vs a current smoker) was also associated with being a daily e-cigarette user ( $\mathrm{aPR}=2.89,95 \% \mathrm{CI}=2.41$ to 3.46 ). Lastly, those who reported using rechargeable or refillable devices were more likely to report daily e-cigarette use compared with those who did not use these devices ( $\mathrm{aPR}=1.95,95 \%$ $\mathrm{CI}=1.44$ to 2.65 and $\mathrm{aPR}=2.10,95 \% \mathrm{CI}=1.75$ to 2.52 , respectively).

\section{Device characteristics, purchasing behaviour and preferred brand among established e- cigarette users}

Among both daily and non-daily established e-cigarette users, 89.5\% reported using an ecigarette that contains nicotine and $67.4 \%$ reported a non-tobacco flavoured regular brand (table 5). The majority of established e-cigarette users $(80.1 \%)$ reported purchasing their products in person, with over half $(56.0 \%)$ purchasing from a tobacco specialty shop/vape shop compared with convenience stores/gas stations $(28.9 \%)$ or other locations $(15.1 \%)$. Daily e-cigarette users were more likely to purchase products online (17.0\%) compared with non-daily users $(11.5 \%)$ ( $\mathrm{p}=0.014)$. Table 5 also displays the top 10 brands reported by daily and non-daily established e-cigarette users. Blu was the most frequently reported brand for both daily $(23.3 \%)$ and non-daily $(41.2 \%)$ users.

\section{DISCUSSION}

The central goal of the current analysis was to describe patterns of e-cigarette use (ie, infrequent, moderate and daily use) in relation to cigarette smoking and other tobacco use. We observed that about four in five e-cigarette users reported less than daily use, with nearly 
half reporting 0-2 days of use in the past month. Additionally, over two-thirds of e-cigarette users reported current cigarette smoking, and about one in six users reported being never cigarette smokers. Consistent with other recent studies, ${ }^{4-6}$ non-daily e-cigarette users were more likely to be young adults (18-24 years) and more likely to currently smoke cigarettes or use other combusted tobacco products compared with daily e-cigarette users.

Consistent with other recent research, ${ }^{57152728}$ being a former smoker versus a current smoker was also associated with daily e-cigarette use. Nearly $89 \%$ of current daily users reported using e-cigarettes as a way of reducing cigarette smoking, and $71.9 \%$ reported using e-cigarettes because 'using e-cigarettes helps people to quit smoking cigarettes.' These findings suggest that for some users, e-cigarettes may have helped them quit smoking and device type may influence the likelihood of this occurring. Wave 1 PATH Study data are cross-sectional and cannot establish temporality or the directionality of such associations; therefore, it is not clear whether daily e-cigarette use facilitated smoking cessation or if cessation occurred prior to daily use of e-cigarettes. Longitudinal data from future waves of the PATH Study will enhance our understanding of these relationships by revealing the timing of transitions between various states of use of e-cigarettes and other tobacco products. Moreover, future waves will permit examination of the stability of use patterns to understand, for instance, what proportion of infrequent users are experimenters who will discontinue use, early-stage initiators who will progress to more frequent use or low-level users who will not remain in their original use state.

These results also highlight an association between device types and patterns of use. In particular, using rechargeable or refillable devices was associated with daily e-cigarette use compared with non-daily use. This suggests that certain e-cigarette design features may be associated with adopting daily use, perhaps facilitating smoking cessation. ${ }^{28}$ Similarly, several studies have suggested that certain device characteristics, such as higher-voltage batteries and heating element resistance, may increase efficacy in nicotine delivery. ${ }^{28}$

An important contribution of the PATH Study to e-cigarette use research is the flexibility it allows for comparison of multiple and more specific definitions of use. Distinguishing ecigarette users according to complex patterns of use is critical, as they are a heterogeneous group: underlying motivations for e-cigarette use differ between infrequent social users compared with daily users seeking an alternative to cigarettes. Early research on e-cigarette awareness and use relied on crude measures such as ever use in one's lifetime or use at least once in the past 30 days, ${ }^{2}$ which likely captured infrequent use due to curiosity and experimentation in addition to stable, frequent use. More recent efforts have focused on refining descriptions of e-cigarette use. ${ }^{61522}$ The present study builds on this growing literature by further disentangling the range of frequency and intensity of use among ecigarette users. This level of detailed information on patterns of use not yet afforded by US national studies provides insight into who, how much and how frequently people are using these products.

\section{Limitations}

The cross-sectional nature of the PATH Study Wave 1 data prevents us from identifying temporal relationships such as the timing of quitting smoking and establishing daily e- 
cigarette use. Given that e-cigarette use is relatively new and the marketplace has rapidly evolved, some respondents may have interpreted questions about product use and characteristics in different ways. For example, some daily users reported they had never used a whole e-cigarette or reported very low lifetime use despite the use of images and detailed descriptions of e-cigarette device types in the PATH Study to facilitate respondents' understanding of the question. Additionally, the cut-point for e-cigarette use categories in this study was based on the distribution of the number of days used out of the past 30 given the limited information available from prospective studies suggesting meaningful cut-points for use patterns. Finally, e-cigarette products are continually evolving; and the products reflected in the US marketplace in 2013-2014 are not necessarily the same as those available at the present time. The PATH Study updates study questionnaires to reflect the marketplace at the time in which each Wave goes into the field. Furthermore, this analysis was limited in its ability to differentiate among currently available device types. It is important to note that use of products with different characteristics (eg, ease, speed and dose of nicotine delivery) may lead to variability in individual patterns of use, including transitions between products. Future research, including future waves of the PATH Study that will include more detailed device type information (eg, use of a tank system and device modifications), should reveal if and how patterns of e-cigarette use vary among users of different device types.

\section{Conclusions}

This analysis adds to the literature on e-cigarette use by describing in greater detail the diverse patterns of e-cigarette use among a nationally representative sample of US adults in 2013-2014. Wave 1 analyses of the PATH Study data suggest that, whereas a majority of ecigarette users also smoke cigarettes, a proportion of e-cigarette users reported former cigarette smoking. Moreover, daily e-cigarette users were more likely to be former smokers compared with non-daily e-cigarette users. The stability of each of these use patterns, their relationship to use of other tobacco products and the impact of these complex behavioural patterns on harmful tobacco-related exposures - and thereby the impact on morbidity and mortality - will ultimately determine the net population health impact of e-cigarettes in the USA.

\section{Acknowledgements}

The authors acknowledge Kimberly Lindsey, Priscilla Callahan-Lyon, Deborah Neveleff and Benjamin Apelberg from the US Food and Drug Administration's Center for Tobacco Products and Karen Messer from the University of California, San Diego, California, USA.

Funding This manuscript is supported with Federal funds from the National Institute on Drug Abuse; National Institutes of Health and the Food and Drug Administration, Departmentof Health and Human Services, under a contract to Westat (Contract No HHSN271201100027C).

\section{REFERENCES}

1. US Food Drug Administration Public Health Focus. Public Health Focus. www.fda.gov/newsevents/ publichealthfocus/(accessed 23 Jan 2016).

2. King BA, Patel R, Nguyen KH, et al. Trends in awareness and use of electronic cigarettes among US adults, 2010-2013. Nicotine Tob Res 2015;17:219-27. [PubMed: 25239961]

3. Regan AK, Promoff G, Dube SR, et al. Electronic nicotine delivery systems: adult use and awareness of the 'e-cigarette' in the USA. Tob Control 2013;22:19-23. [PubMed: 22034071] 
4. McMillen RC, Gottlieb MA, Shaefer RM, et al. Trends in electronic cigarette use among U.S. adults: use is increasing in both smokers and nonsmokers. Nicotine Tob Res 2015;17:1195-202. [PubMed: 25381306]

5. Schoenborn CA, Gindi RM. Electronic cigarette use among adults: United States, 2014 Hyattsville, MD: National Center for Health Statistics, 2015. NCHS Data Brief no. 217.

6. Delnevo CD, Giovenco DP, Steinberg MB, et al. Patterns of electronic cigarette use among adults in the United States. Nicotine Tob Res 2016;18:715-9. [PubMed: 26525063]

7. McMillen R, Maduka J, Winickoff J. Use of emerging tobacco products in the United States. J Environ Public Health 2012;2012:1-8.

8. Cobb NK, Abrams DB. The FDA, e-cigarettes, and the demise of combusted tobacco. N Engl J Med 2014;371:1469-71. [PubMed: 25317866]

9. Levy DT, Cummings KM, Villanti AC, et al. A framework for evaluating the public health impact of e-cigarettes and other vaporized nicotine products. Addiction 2017;112:1-7.

10. McNeil A, Brose LS, Calder R, et al. E-cigarettes: an evidence update. A report commissioned by Public Health England. PHE publications gateway number:2015260 www.gov.uk/government/ uploads/system/uploads/attachment_data/file/457102/ Ecigarettes_an_evidence_update_A_report_commissioned_by_Public_Health_England_FINAL.p df.

11. Hartmann-Boyce J, McRobbie H, Bullen C, et al. Electronic cigarettes for smoking cessation. Cochrane Database Syst Rev 2016;9.

12. Pulvers K, Hayes RB, Scheuermann TS, et al. Tobacco use, quitting behavior, and health characteristics among current electronic cigarette users in a National Tri-Ethnic adult stable smoker sample. Nicotine Tob Res 2015;17:1085-95. [PubMed: 25385875]

13. Christensen T, Welsh E, Faseru B. Profile of e-cigarette use and its relationship with cigarette quit attempts and abstinence in Kansas adults. Prev Med 2014;69:90-4. [PubMed: 25230365]

14. Zhu SH, Sun JY, Bonnevie E, et al. Four hundred and sixty brands of e-cigarettes and counting: implications for product regulation. Tob Control 2014;23(Suppl 3):iii3-9. [PubMed: 24935895]

15. Biener L, Hargraves JL. A longitudinal study of electronic cigarette use among a population-based sample of adult smokers: association with smoking cessation and motivation to quit. Nicotine Tob Res 2015;17:127-33. [PubMed: 25301815]

16. Agaku IT, King BA, Husten CG, et al. Tobacco product use among adults-United States, 20122013. MMWR Morb Mortal Wkly Rep 2014;63:542-7. [PubMed: 24964880]

17. Schmidt L, Reidmohr A, Harwell TS, et al. Prevalence and reasons for initiating use of electronic cigarettes among adults in Montana, 2013. Prev Chronic Dis 2014;11:E204. [PubMed: 25412027]

18. Pepper JK, Ribisl KM, Emery SL, et al. Reasons for starting and stopping electronic cigarette use. Int J Environ Res Public Health 2014;11:10345-61. [PubMed: 25286168]

19. Dockrell M, Morrison R, Bauld L, et al. E-cigarettes: prevalence and attitudes in Great Britain. Nicotine Tob Res 2013;15:1737-44. [PubMed: 23703732]

20. Gravely S, Fong GT, Cummings KM, et al. Awareness, trial, and current use of electronic cigarettes in 10 countries: findings from the ITC project. Int J Environ Res Public Health 2014;11:11691-704. [PubMed: 25421063]

21. Gallus S, Lugo A, Pacifici R, et al. E-cigarette awareness, use, and harm perceptions in Italy: a national representative survey. Nicotine Tob Res 2014;16:1541-8. [PubMed: 25082832]

22. Giovenco DP, Lewis MJ, Delnevo CD. Factors associated with e-cigarette use: a national population survey of current and former smokers. Am J Prev Med 2014;47:476-80. [PubMed: 24880986]

23. Amato MS, Boyle RG, Levy D. How to define e-cigarette prevalence? Finding clues in the use frequency distribution. Tob Control 2016 25e1:e24-;25.

24. Hyland A, Ambrose BK, Conway KP, et al. Design and methods of the Population Assessment of tobacco and Health (PATH) Study. Tob Control 2016:tobaccocontrol-2016-052934.

25. Zou G A modified poisson regression approach to prospective studies with binary data. Am J Epidemiol 2004;159:702-6. [PubMed: 15033648] 
26. Johnson CL, Paulose-Ram R, Ogden CL, et al. National Health and Nutrition Examination survey: analytic guidelines, 1999-2010. National Center for Health Statistics. Vita Health Stat 2013.

27. Brose LS, Hitchman SC, Brown J, et al. Is the use of electronic cigarettes while smoking associated with smoking cessation attempts, cessation andreduced cigarette consumption? A survey with a 1-year follow-up. Addiction 2015;110:1160-8. [PubMed: 25900312]

28. Hitchman SC, Brose LS, Brown J, et al. Associations between e-cigarette type, frequency of use, and quitting smoking: findings from a Longitudinal Online Panel Survey in Great Britain. Nicotine Tob Res 2015;17:1187-94. [PubMed: 25896067]

29. Shihadeh A, Eissenberg T. Electronic cigarette effectiveness and abuse liability: predicting and regulating nicotine flux. Nicotine Tob Res 2015;17:158-62. [PubMed: 25180079]

30. Yingst JM, Veldheer S, Hrabovsky S, et al. Factors associated with electronic cigarette users' device preferences and transition from first generation to dvanced generation devices. Nicotine Tob Res 2015;17:1242-6. [PubMed: 25744966]

31. Grana RA, Popova L, Ling PM. A longitudinal analysis of electronic cigarette use and smoking cessation. JAMA Intern Med 2014;174:812-3. [PubMed: 24664434]

32. Popova L, Ling PM. Alternative tobacco product use and smoking cessation: a national study. Am J Public Health 2013;103:923-30. [PubMed: 23488521] 


\section{What this paper adds}

- $\quad$ Research indicates that prevalence of ever and current e-cigarette use among adults in the USA is highest among recent former and current smokers. Although several US population-based surveys have reported on prevalence of ever and current e-cigarette use in the context of cigarette smoking, a gap remains regarding detailed product information (eg, device characteristics), purchasing behaviour and motivations for use. Our study provides details on these characteristics that have not been captured by other US national crosssectional surveys to date.

- The majority of e-cigarette users in this study reported less than daily use, with nearly half reporting 0-2 days of use in the past month. Compared with non-daily use, daily use was associated with using rechargeable or refillable devices as well as being a former smoker. 


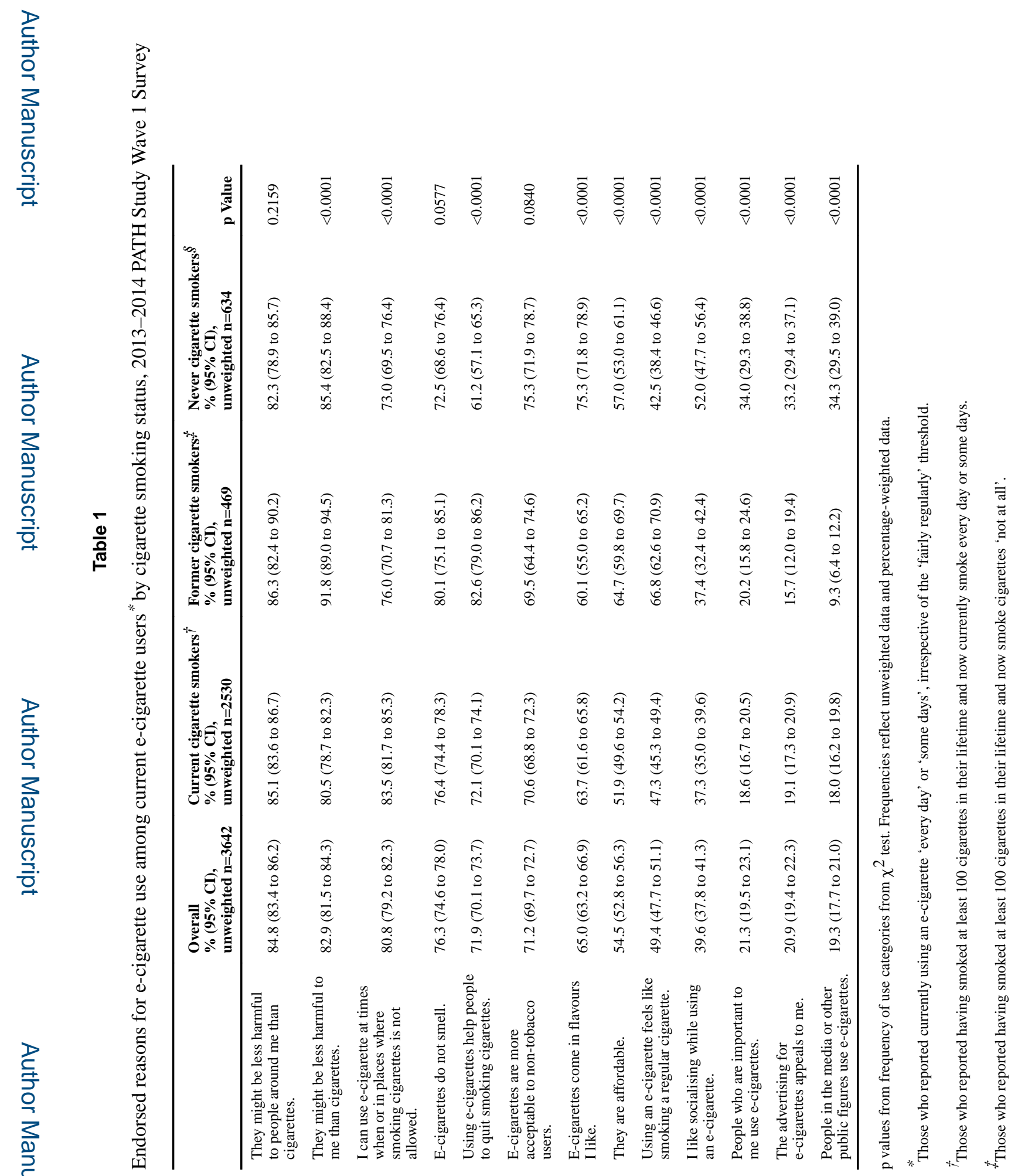

Tob Control. Author manuscript; available in PMC 2019 August 14. 


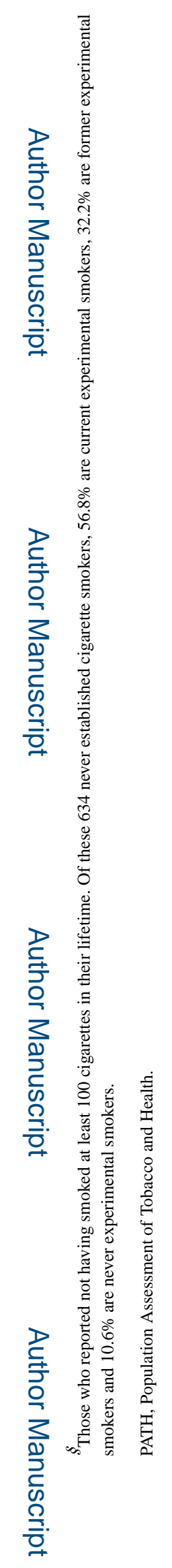

Tob Control. Author manuscript; available in PMC 2019 August 14. 


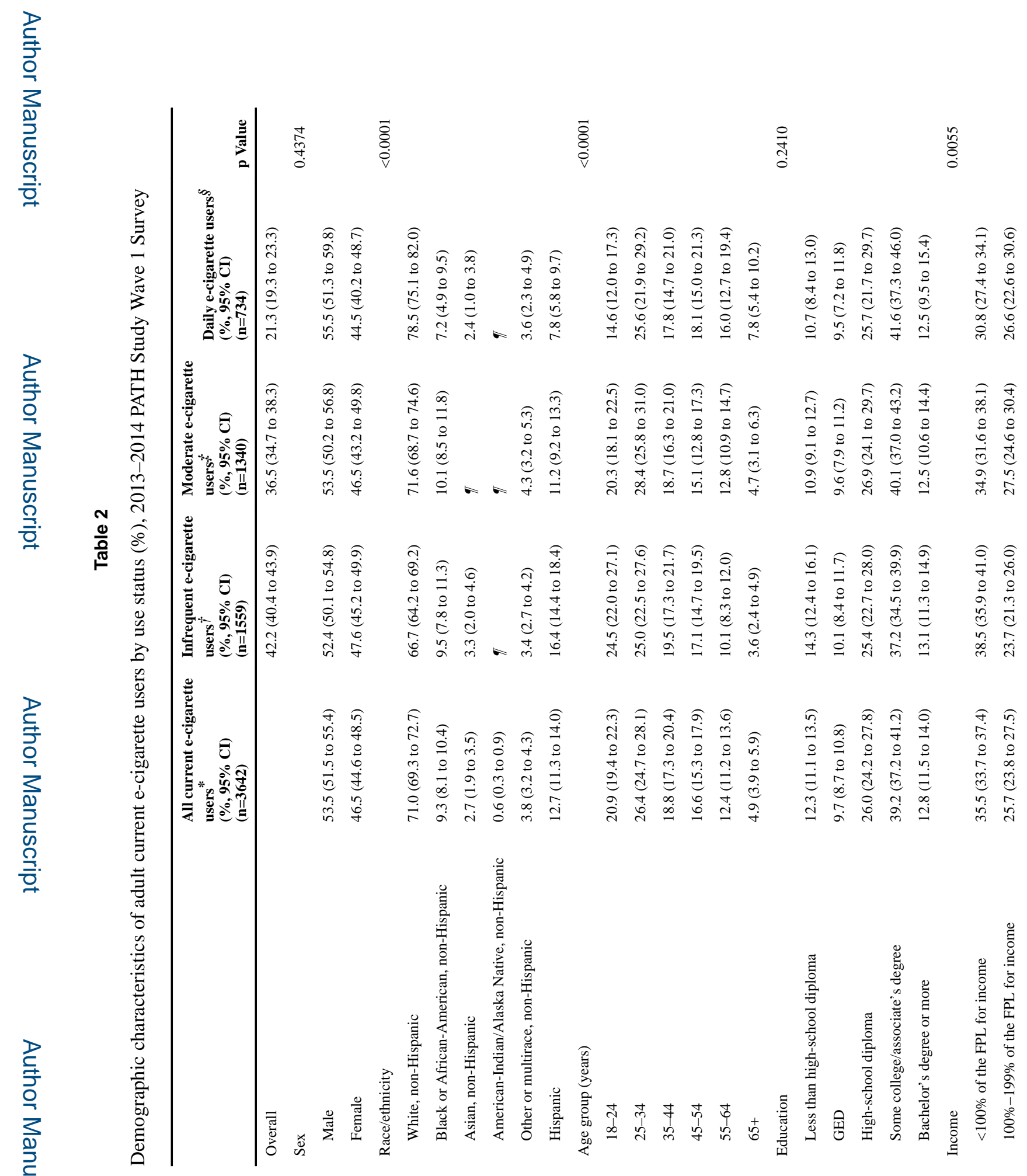

Tob Control. Author manuscript; available in PMC 2019 August 14. 


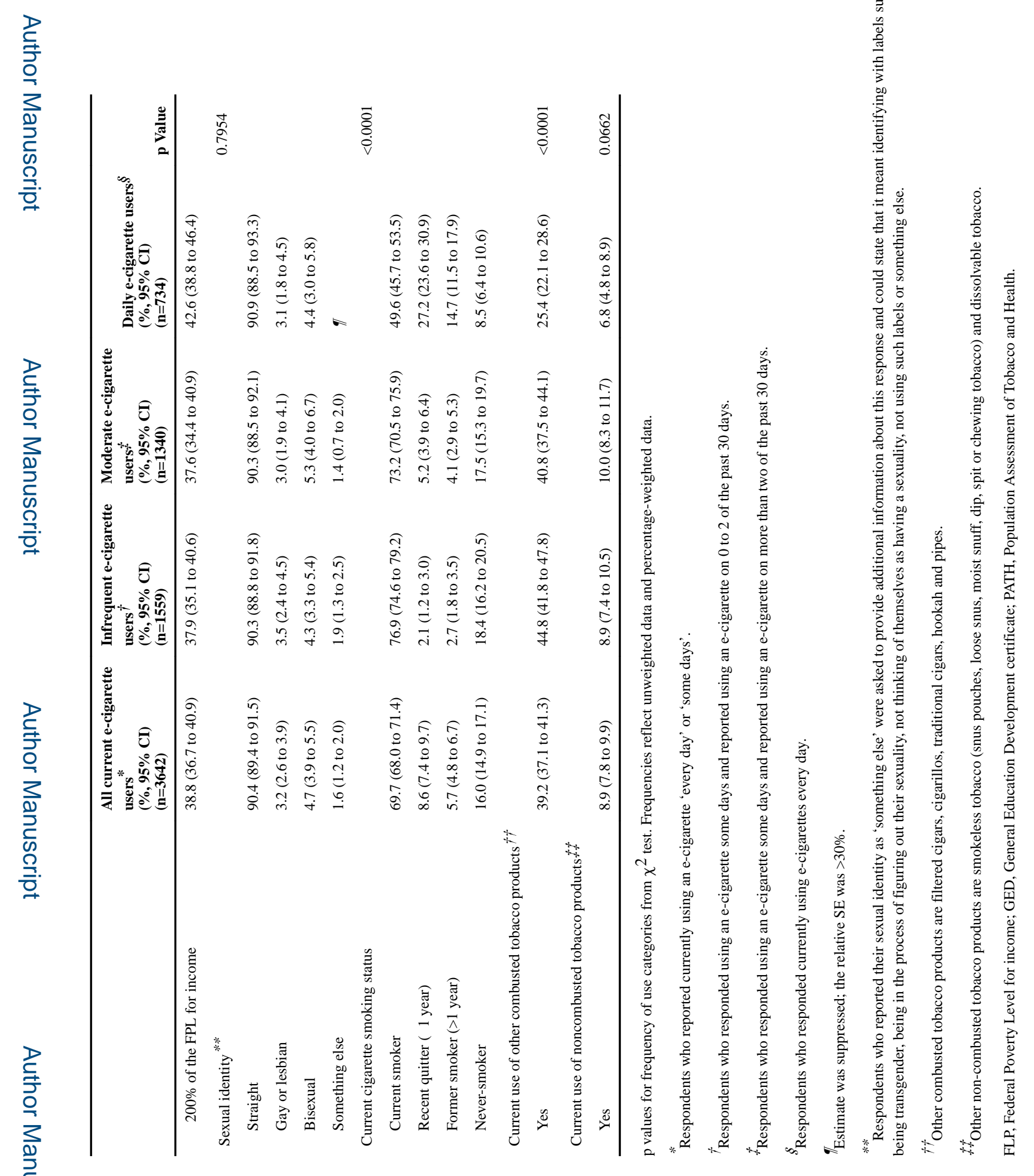




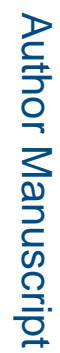

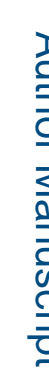

疍

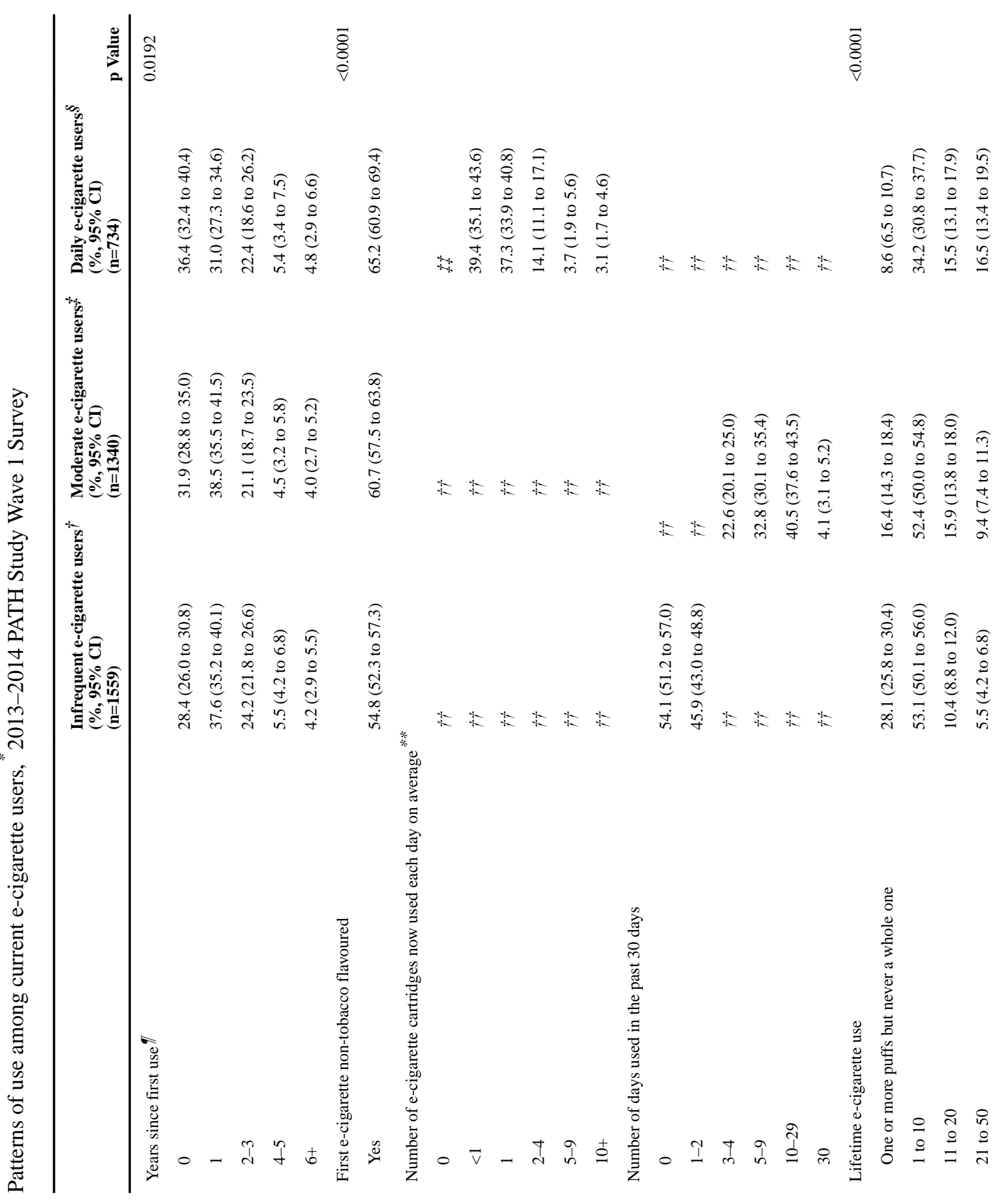

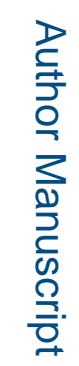

Tob Control. Author manuscript; available in PMC 2019 August 14. 


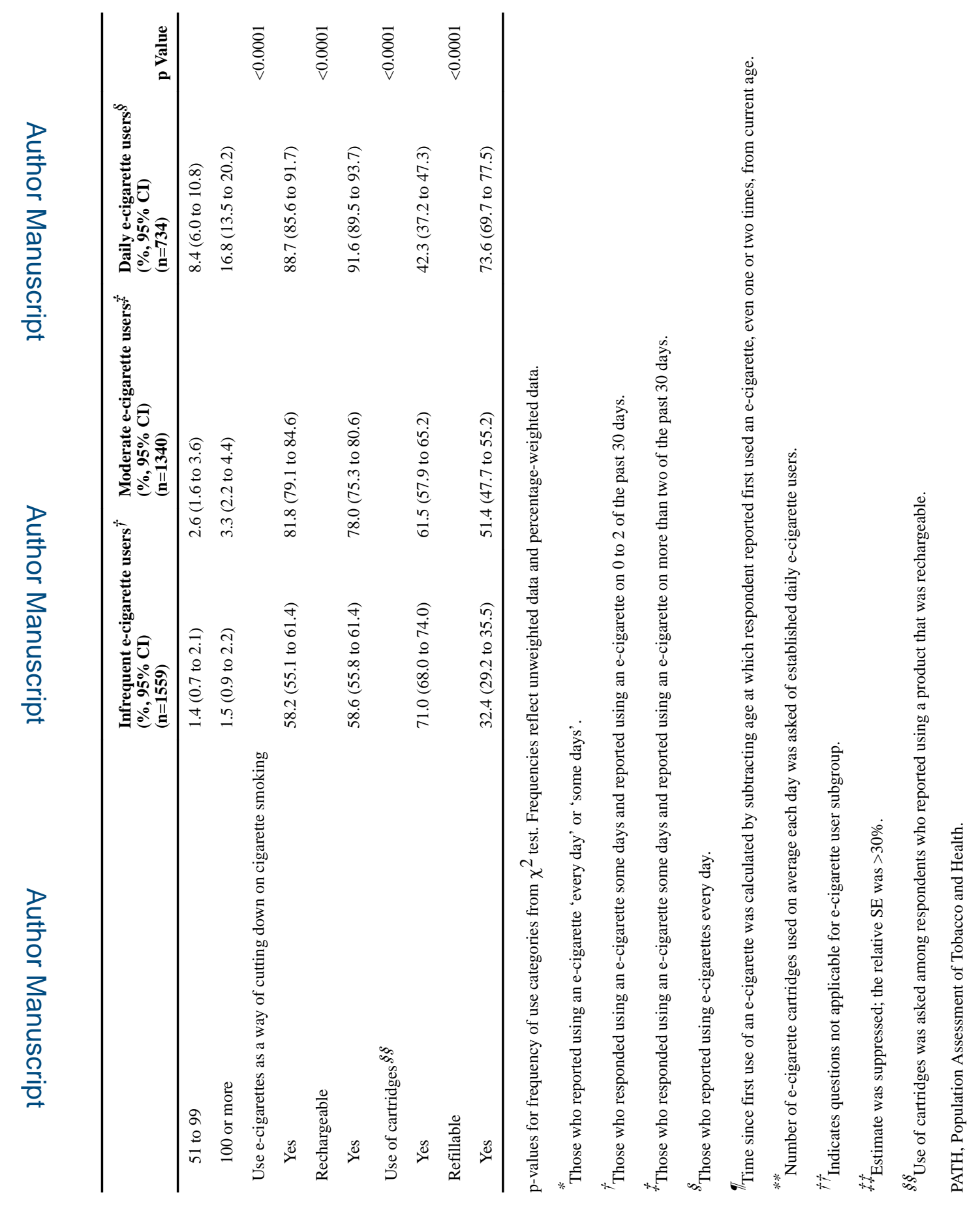

로을

Tob Control. Author manuscript; available in PMC 2019 August 14. 


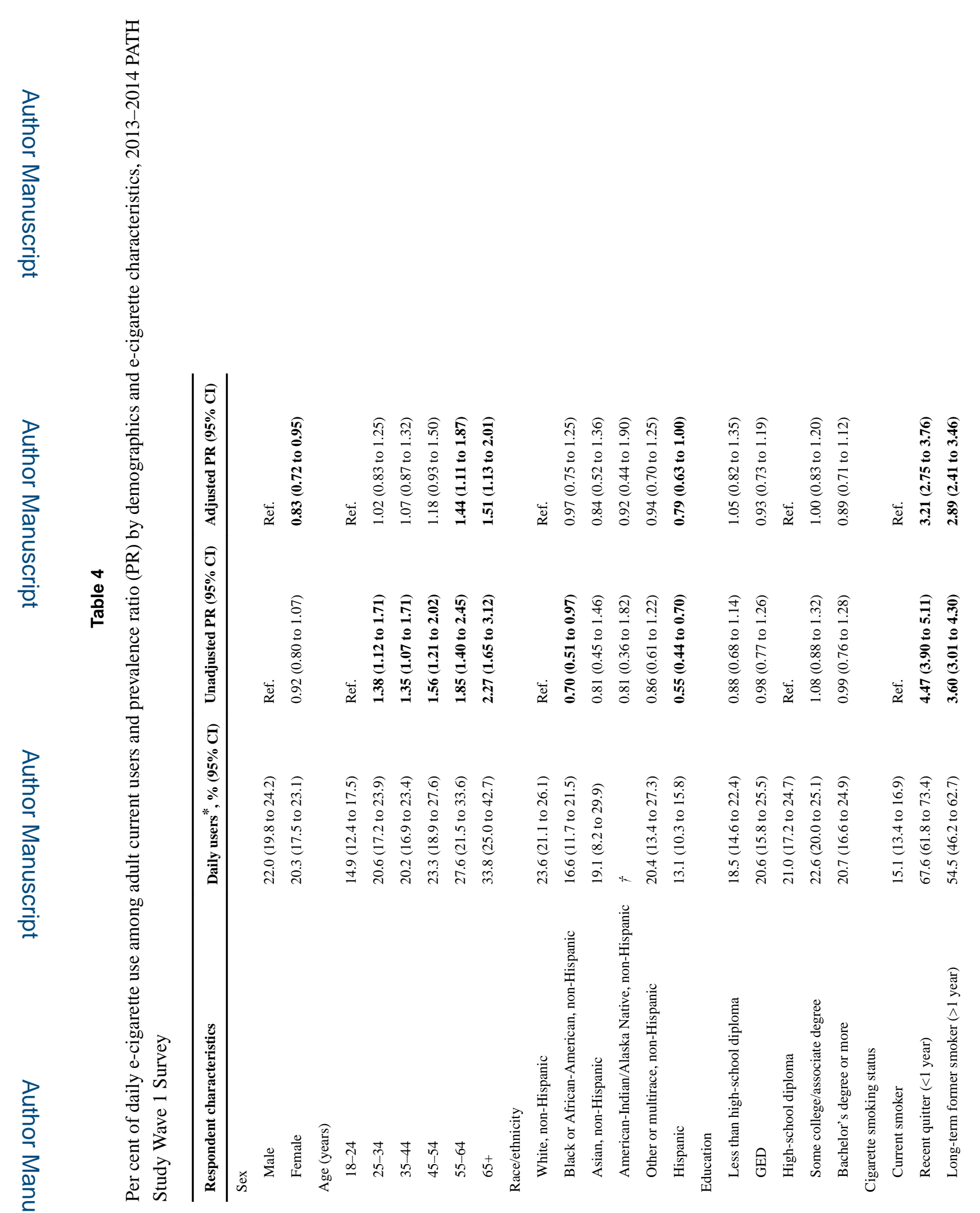




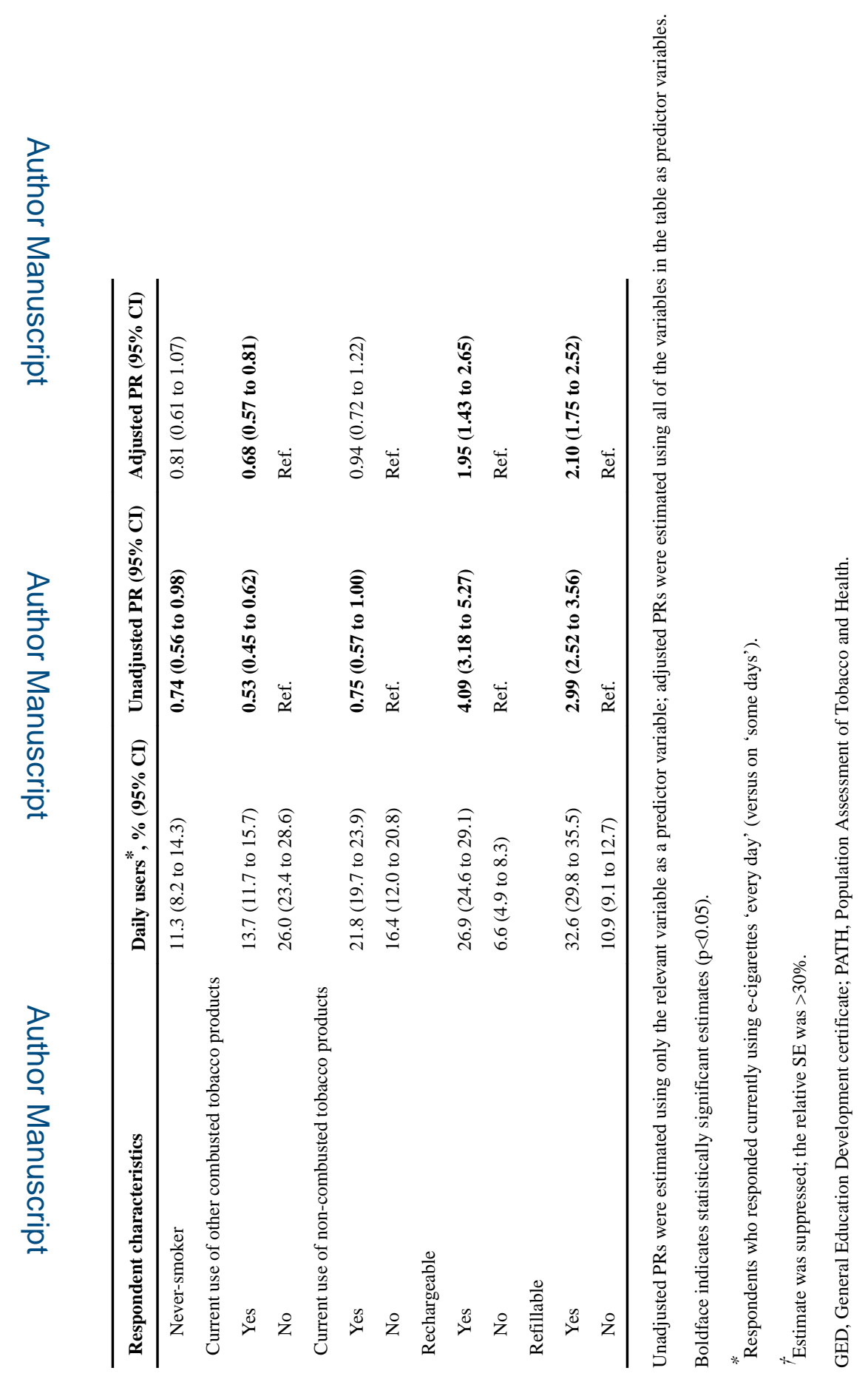

로을

Tob Control. Author manuscript; available in PMC 2019 August 14. 


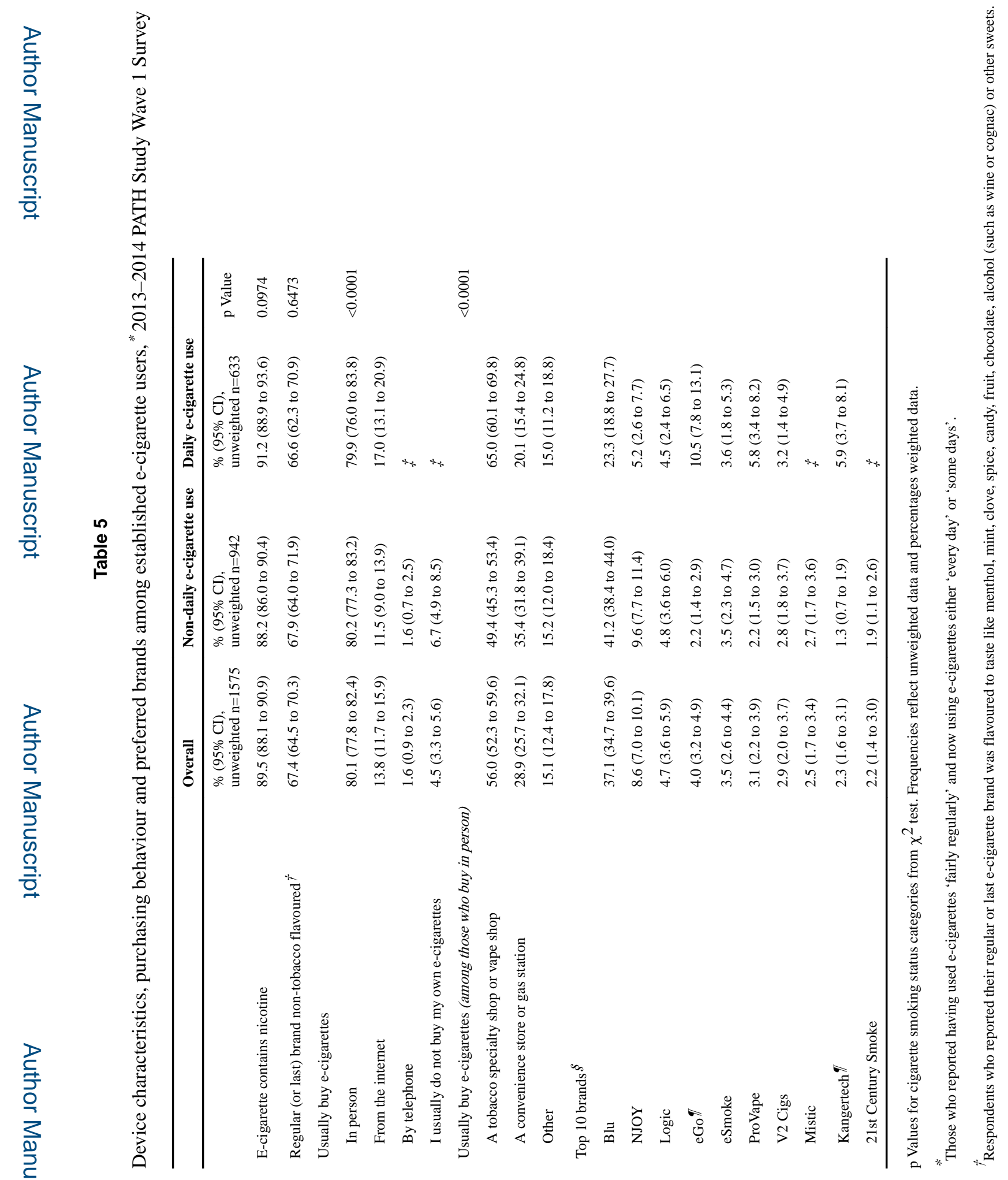

Tob Control. Author manuscript; available in PMC 2019 August 14. 


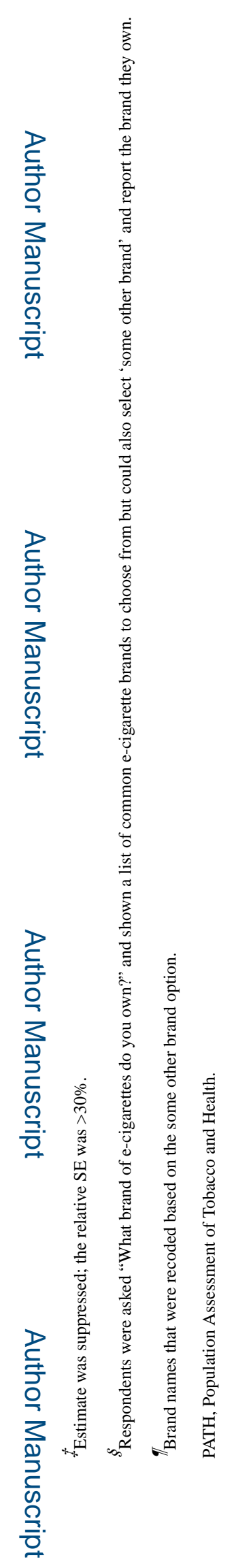

